\title{
What is the effect of phasing out long-chain per- and polyfluoroalkyl substances on the concentrations of perfluoroalkyl acids and their precursors in the environment? A systematic review protocol
}

Magnus Land ${ }^{1 *}$, Cynthia A de Wit², lan T Cousins², Dorte Herzke ${ }^{3}$, Jana Johansson² and Jonathan W Martin ${ }^{4}$

\begin{abstract}
Background: There is a growing concern in Sweden and elsewhere that continued emissions of per- and polyfluoroalkyl substances (PFASs) may cause environmental as well as human health effects. PFASs are a broad class of man-made substances that have been produced and used in both commercial products and industrial processes for more than 60 years. Although the production and use of some PFASs has been phased-out in some parts of the world, it is not known what effect these actions to date have had on PFAS concentrations in the environment. Owing to the wide diversity of PFASs, it is difficult to generalize their properties, environmental fate and production histories. However, the strength and stability of the C-F bond renders the perfluoroalkyl moieties resistant to heat and environmental degradation. Several PFASs are now occurring even in very remote areas in large parts of the world, but the environmental transport and fate of substances within this group is not well understood. A systematic review may be able to determine whether the concentrations of these substances in different environments are changing in any particular direction with time, and whether the phase-outs have had any effects on the concentration trends.

Methods: Searches for primary research studies reporting on temporal variations of PFAS concentrations in the environment will be performed in the scientific literature as well as in other reports. Relevant samples include both abiotic and biological samples including humans. No particular time, document type, language or geographical constraints will be applied. Two authors will screen all retrieved articles. Double screening of about $10 \%$ of the articles will be performed by all authors at both title/abstract and full-text levels. Kappa tests will be used to test if the screening is consistent. Relevant articles will be critically appraised by four authors (double checking of $25 \%$ of the articles). Quality assessment will focus on selection bias, dating of samples, sample integrity and analytical procedures. Data synthesis will be based on statistical analysis of temporal concentration trends.
\end{abstract}

Keywords: Perfluoroalkyl acids, Perfluoroalkane acids, PFOA, PFOS, Temporal trends, Phase-out, Source, Emission, Environmental fate, Regulation, Concentration

\footnotetext{
* Correspondence: magnus.land@eviem.se

1 Mistra EviEM, The Royal Swedish Academy of Sciences, Box 50005, SE-104 05

Stockholm, Sweden

Full list of author information is available at the end of the article
} 


\section{Background}

Per- and polyfluoroalkyl substances (PFASs) are a broad class of man-made substances that have been produced and used in commercial products and industrial processes for over 60 years [1]. For example, PFASs have been used in water-, soil-, and stain-resistant coatings for clothing, leather, upholstery, and carpets; oil-resistant coatings for food contact paper; aviation hydraulic fluids; fire-fighting foams; paints, adhesives, waxes, polishes, and other products; and industrially as surfactants, emulsifiers, wetting agents, additives, and coatings [2-7]. The perfluoroalkyl moieties $\left(\mathrm{C}_{\mathrm{n}} \mathrm{F}_{2 \mathrm{n}+1}-\right)$ of PFAS molecules are both hydrophobic and lipophobic [2], and the extreme strength and stability of the C-F bond [8] renders the perfluoroalkyl moieties resistant to heat and environmental degradation processes. Nevertheless, owing to the wide diversity of PFASs (i.e. chain-lengths, molecular weight, degree and pattern of fluorination, presence of polar functional groups), it is difficult to generalize their properties, environmental fate, and production histories [9]. For purposes of this document we have separated the discussion into two broad categories, which will be the focus of our study:

1) Perfluoroalkyl carboxylic acids (PFCAs) and their precursors

2) Perfluoroalkane sulfonic acids (PFSAs) and their precursors

We decided to focus on PFCAs and PFSAs because these are by far the two most widely studied PFAS classes, and multiple temporal trend datasets were known to be available. PFCAs and PFSAs are well studied due to their persistence and ubiquity in the environment and biota as well as their potentially harmful effects (see brief review below). We use the terminology recommended by Buck et al. [9] throughout this document. A list of abbreviations used in this protocol is provided in Additional file 1: List of abbreviations.

\section{Perfluoroalkyl carboxylic acids (PFCAs) and their precursors}

PFCAs occur in the environment due to emission from intentional manufacturing, as impurities in commercial products containing other PFASs, or as environmental degradation products of other PFASs [3-5]. The PFCA manufactured in the largest quantity, perfluorooctanoic acid (PFOA), was produced mainly by electrochemical fluorination (ECF) by 3M until 2002 (3M had $>80 \%$ of global market) and was primarily used as a processing aid (emulsifier) in the manufacture of polytetrafluoroethylene (PTFE). The ECF manufacturing process produces a mixture of linear (70\%) and branched (30\%) isomers. After $3 \mathrm{M}$ phased-out the production of PFOA in 2002, other companies continued to manufacture PFOA mainly through the telomerization process which produces only linear isomers. For a thorough description of the sources of PFCA homologues and their precursors see the reviews of Prevedouros et al. [3], Wang et al. [4] and Wang et al. [5].

The most common PFCA measured in the abiotic environment is PFOA. Once present in the environment, the PFCAs have no significant known mode of environmental degradation and are thus highly persistent. For example, in wastewater treatment plants PFOA did not degrade, but actually increased in the outflow owing to degradation of unidentified PFCA precursors [10]. Engineering solutions using oxidants and catalysts have been developed to degrade PFCAs on a small scale in the lab. For example, UV photo-oxidation with indium oxide $\left(\operatorname{In}_{2} \mathrm{O}_{3}\right)$ as the catalyst is effective for PFOA degradation [11], but these processes will not occur in the natural environment.

PFCAs have an acid dissociation constant (pKa) in the range $0-1$ and are thus completely dissociated anions in environmental media which typically have $\mathrm{pH}$ above 4 [12-15]. Once discharged into the environment, unlike typical persistent organic substances, the majority of PFCAs do not sorb appreciably on particles but are instead present mainly in the dissolved phase in surface waters. Sorption to the organic fraction of particles increases with the length of the perfluoroalkyl chain [16], but the organic-carbon water partition coefficient $\left(\mathrm{K}_{\mathrm{OC}}\right)$ of a long-chain PFCA (C8 and higher) such as PFOA [16] is still orders of magnitude lower than for typical persistent organic substances. Their low sorption and high prevalence in surface waters is evident from field investigations demonstrating that PFOA in biosolids applied to agricultural land can be re-mobilized by rainfall [17], and it has been shown that the sediment/water partition coefficient is low (PFOA K $\mathrm{OC}=2.4 \pm 0.12 \mathrm{~cm}^{3} \mathrm{~g}^{-1}$ ) [18]. As a result, PFCAs can be transported long distances by rivers and ocean currents and now occur in the open marine environment, even in the remote Northern Atlantic, Pacific, and Arctic Oceans [19,20]. PFCAs can also be detected at low concentration in the ambient atmosphere, where they may be directly emitted [21], and/or formed in situ by oxidation of semivolatile PFCA-precursors such as the fluorotelomer alcohols (FTOH) [22].

Overall, the direct and indirect sources of PFCAs to the environment are various, and the relative importance of each source is temporally variable, PFCA-specific and not well quantified. For a given environmental medium in a given region, it is challenging to determine the source contribution to the PFCA contamination profile because in addition to uncertainties in the relative importance of multiple source types, the contamination 
profile will be transformed as a result of differences in the fate and transport of the individual PFCAs after release. The relative importance of atmospheric versus marine transport of PFCAs, of direct atmospheric emission versus atmospheric oxidation of PFCA-precursors, or of telomerization versus electrochemical manufacturing of PFCAs and their precursors is the subject of much recent and ongoing research. For PFCAs and their precursors, the perfluoroalkyl chain-length can have a considerable effect on their environmental fate and partitioning $[16,23]$.

Occurring to a large extent in the dissolved phase in surface waters, PFCAs are relatively bioavailable compared to typical hydrophobic organic substances. PFCAs have been detected in numerous biological samples, e.g., fish [24], bird eggs [25-27], invertebrates, reptiles and marine mammals including polar bear [28], as well as humans $[29,30]$.

Bioaccumulation has been shown to occur in mammals and birds, increasing with perfluoroalkyl chain length [31]. A limited number of field-Biomagnification Factors (BMFs) and Trophic Magnification Factors (TMFs) are available for long-chain PFCAs (and perfluorooctane sulfonate, PFOS) and they provide evidence that biomagnification of these substances takes place. Tomy et al. [32] reported bioaccumulation of perfluoroalkyl acids (PFAAs, including PFCAs and PFSAs) occurring in Arctic marine food webs. Kelly et al. [33] compared different parts of the marine food web, showing that the TMF is below one in the case of piscivorous food webs if air breathing organisms are excluded but becomes larger than one if air breathing organisms are taken into account. Loi et al. [34] observed trophic magnification for long-chain PFCAs (perfluorodecanoic acid (PFDcA), perfluoroundecanoic acid (PFUnA), perfluorododecanoic acid (PFDoA)) and PFOS in a subtropical food web. In freshwater systems, Martin et al. [35] found BMFs of long-chain PFCAs to vary from 0.4 to 3.4 between lake trout and prey organisms in Canada (PFOA had the lowest BMF and PFUnA the highest). In terrestrial samples, Muller et al. [36] reported BMFs and TMFs from caribou to lichen for PFAAs. Highest BMFs of 75 and 46 were found for PFDcA and PFUnA, respectively. TMFs of the food chain wolf caribou - lichen varied between 2.4 and 7.1 for all longchain PFAAs with PFDcA and PFOS showing the highest TMFs of 7.1 and 6.7 respectively. In summary, BMFs and TMFs above 1 indicate trophic biomagnification for PFAAs with a perfluoroakyl chain length containing 8 or more perfluorinated carbons in the terrestrial and freshwater ecosystems studied. Some short-chain (e.g. butane- and hexane-based) alternatives also appear to be persistent but to not bioaccumulate to the same extent, as they are excreted rapidly from the organisms studied [37].
PFCAs are not acutely toxic based on standard toxicity endpoints. However, they have been reported to have endocrine disrupting properties [38-40]. PFAAs are structurally similar to natural long-chain fatty acids and may displace them in biochemical processes and at receptors, such as peroxisome proliferator-activated receptor alpha (PPAR $\alpha$ ) and the liver-fatty acid binding protein (L-FABP). PFCAs, particularly the long-chain PFOA, PFNA (perfluorononanoic acid) and PFDcA but not the short-chain PFHxA (perfluorohexanoic acid), are highly potent peroxisome proliferators in rodent livers and affect mitochondrial, microsomal, and cytosolic enzymes and proteins involved in lipid metabolism [41-44]. PFCAs cause hepatomegaly in rodents [42] which is an indicator for hepatotoxicity. Perfluorobutanoic acid (PFBA) has a less pronounced effect on indicators of peroxisome proliferation [41].

Starting in 2000, various actions were undertaken by industry and regulators to reduce the release of PFCAs and precursors. In 2000, 3M announced a global phaseout by 2002 of its production of products based on perfluoroalkyl chains containing 6,8 and 10 carbons, including PFOA [45]. In 2006, eight major PFCA, fluoropolymer and fluorotelomer manufacturers joined the US EPA 2010/15 Stewardship Program to work towards the elimination of long-chain PFCAs and their precursors from emissions and products by 2015 [46]. On the regulatory front, $\mathrm{PFOA}$, its ammonium salt ammonium perfluorooctanoate (APFO), and C11-C14 PFCAs were included in the Candidate List of Substances of Very High Concern under the European chemicals regulation, REACH [47]. Although long-chain PFCAs are being stepwise phased out by the major manufacturers and heavily regulated in Japan, Western Europe and the United States (US) $[45,46,48]$, new manufacturers (largely in continental Asia) have begun to produce long-chain PFCAs and their precursors. As a result of the phase out of many long-chain PFASs in recent years, many alternative fluorinated products have been introduced [49] and these new, alternative industrial processes and products have resulted in new sources of PFCAs and other fluorinated substances. For example, there has likely been increasing emissions of PFHxA due to the increasing use of sidechain polymers based on 6:2 FTOHs in surface treatment products [49]. A time-series of key production events and regulatory actions for PFCAs is shown in Figure 1.

\section{Perfluoroalkane sulfonic acids (PFSAs) and their precursors}

Similar to PFCAs, sources of PFSAs include release during manufacture and use of the PFSAs as well as from the degradation of various precursor substances $[6,50]$. Commercial scale manufacture of perfluorooctane sulfonyl fluoride (POSF)-based products began by $3 \mathrm{M}$ in 


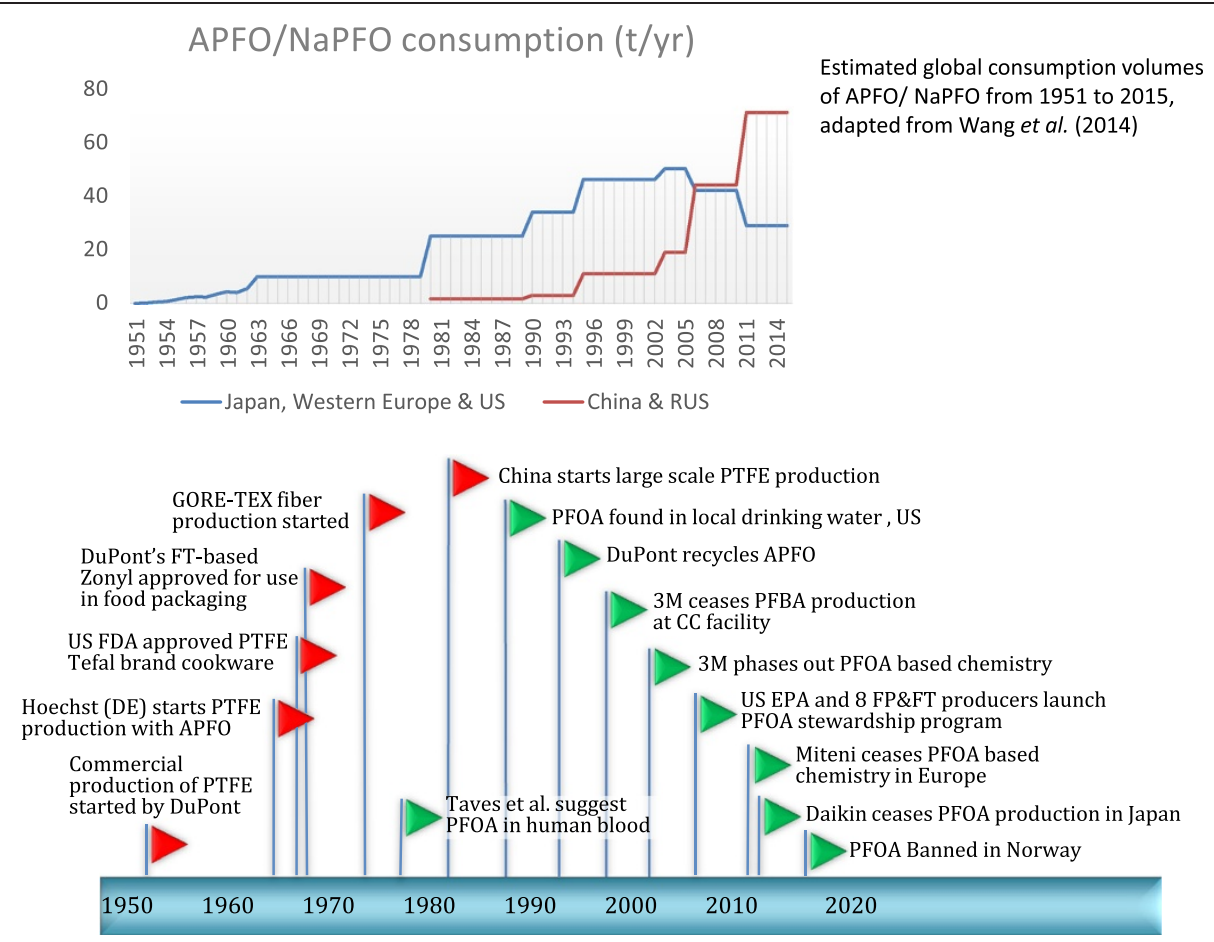

Figure 1 Timeline of production, commercialization and legislation of PFCAs. APFO (ammonium perfluorooctanoate) and NaPFO (sodium perfluorooctanoate) are salts of PFOA. Red flags represent events and actions that may have resulted in increased concentrations in the environment. Green flags represent important findings and phase-outs that may result in decreased concentrations in the environment.

the late 1950s with product lines based on N-methyl perfluorooctane sulfonamido ethanols (N-MeFOSEs) used in surface treatment applications (e.g., carpets, upholstery and textiles) and in the late 1960s product lines based on $\mathrm{N}$-ethyl perfluorooctane sulfonamido ethanols (NEtFOSEs) were introduced for use in paper and board packaging applications. PFOS and various salts were manufactured for direct use in a variety of products (e.g. aqueous film-forming foams (AFFFs) for firefighting and mist suppressants in acids baths used for metal plating (for a more complete list of uses of PFOS and its salts see Paul et al. [6])). Commercial use of PFOS and its salts first started around 1970 [6]. Uses and emissions of PFSAs and their precursors have been estimated to have steadily risen after manufacture started until a maximum usage was reached at the end of the $20^{\text {th }}$ century [6]. PFSAs and precursors have been manufactured by ECF which produced a mixture of linear (70\%) and branched (30\%) isomers [51]. 3M also historically made products based on perfluorohexane and perfluorodecane sulfonyl fluoride [50]. For a more thorough description of sources of PFSA homologues and their precursors see the reviews of Paul et al. [6] and Armitage et al. [50].

PFOS and other PFSAs are widely distributed in the global environment [52-56], biota [57-62] and humans $[29,63-70]$. Due to the dominance of POSF-based products historically, PFOS is usually found to be the most abundant PFSA. PFSAs are stronger acids than PFCAs with pKa's $<0$ and are thus fully dissociated anions in environmental media [15]. Although properties vary with chain-length, the environmental fate and bioaccumulation behavior of PFSAs is broadly similar to that of PFCAs; PFSAs are persistent, are mostly distributed to surface waters [50], bind weakly to organic phases [16] compared to other persistent organic substances, are shown to bioaccumulate in the laboratory [71,72] and biomagnify in food webs [73,74]. Also similar to PFCAs, the global distribution of PFSAs is governed by a combination of direct release and transport as well as release and transport of precursors that subsequently degrade to PFSAs [50]. One difference in behavior is that PFSAs with perfluoroalkyl chains of the same length tend to sorb [16] and bioaccumulate $[31,75,76]$ more strongly than PFCAs, [31,75,77] which is an effect of the different anionic head groups. Consequently, PFSAs with perfluoroalkyl chain lengths of C6 (i.e. perfluorohexane sulfonate) and higher are considered to be long-chain (http://www.oecd.org/ehs/pfc/), whereas for PFCAs those with perfluoroalkyl chain lengths of C8 (i.e. PFOA) and higher are considered to be long-chain.

In 2000 the major manufacturer of PFOS in the US (3M) announced their plan to cease the production of 
$\mathrm{C}_{6}, \mathrm{C}_{8}$ and $\mathrm{C}_{10}$ perfluoroalkane sulfonyl fluoride (PASF)based products and completed the phase out in 2002 [78]. In 2006 the EU adopted a Marketing and Use Directive (2006/122/EC) that bans the use of PFOS in semi-finished products (maximum content of PFOS: $0.005 \%$ by weight) as of summer 2008. In 2009, PFOS (and related substances derived from the POSF) were listed under Annex B (restriction of production and use) of the Stockholm Convention on Persistent Organic Pollutants. After 2000, China filled the gap created between global supply and demand of PFOS (and related products) caused by the $3 \mathrm{M}$ phase out. According to Zhang et al. [79], the production volume of PFOS increased from $30 \mathrm{t}$ in 2002 to $247 \mathrm{t}$ in 2006. Since then, caused by international legislation to restrict or eliminate PFOS production, the production volume of PFOS has declined to about $100 \mathrm{t} / \mathrm{y}$ in 2008.

Since 2003, 3M has commercialized new surface treatment products based on perfluorobutane sulfonate (PFBS, C4 sulfonate) [80]. Although likely to be as environmentally persistent as PFOS, PFBS is thought to be less bioaccumulative and toxic. It is likely that PFBS is released during manufacture and use of 3M's new surface treatment products and that increasing usage will lead to increasing levels in the environment. Evidence for the environmental release of PFBS can be found from its reported presence in the aquatic environment [81,82], biota [83] and humans [84]. A time-series of key production events and regulatory actions for PFSAs is shown in Figure 2.

\section{Genesis of the systematic review}

A systematic review on PFASs in the environment was originally suggested by the Swedish Chemicals Agency at a general stakeholder meeting arranged by Mistra EviEM in January 2012, to which a broad spectrum of organizations were invited and encouraged to suggest topics for systematic reviews. The Swedish Chemicals Agency is responsible for the Swedish environmental quality objective "A non-toxic environment" [85]. There is a growing concern in Sweden that continued discharges of PFASs may cause environmental as well as human health effects. Borg and Håkansson [86] performed a risk assessment consisting of an exposure assessment based on Swedish biomonitoring data, a hazard assessment with toxicological data from studies on mammals, birds and fish, and a risk characterization. The result of the environmental risk characterization indicated a cause for concern for seals and otters for hepatotoxicity and reproductive toxicity. For reproductive toxicity in birds, a cause for concern was indicated for PFOS where the highest level in peregrine falcons eggs (sampled in 2006) exceeded the toxic effect level identified in a study by Molina et al. [87]. Regarding human health effects, at least three different municipal drinking water plants in Sweden have been shut down due to high concentrations

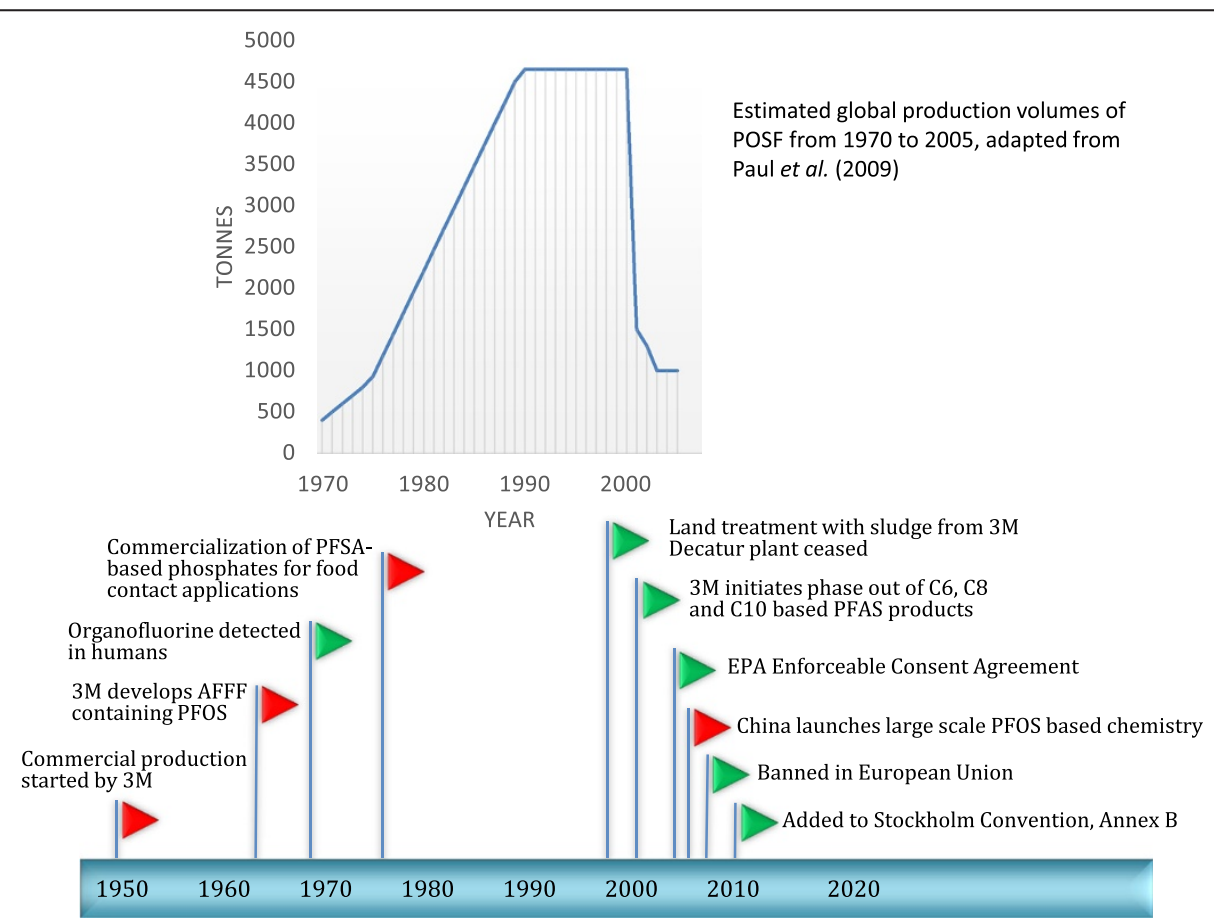

Figure 2 Timeline of production, commercialization and legislation of PFSAs. POSF (perfluorooctane sulfonyl fluoride) is the major raw material used to manufacture PFOS. Red flags represent events and actions that may have resulted in increased concentrations in the environment. Green flags represent important findings and phase-outs that may result in decreased concentrations in the environment. 
of PFOS in the groundwater source. It is believed that the high concentrations are caused by spills of fire-fighting foam at adjacent airports or firefighting training sites.

A specific question regarding PFASs was not formulated at the general stakeholder meeting arranged by Mistra EviEM, but it emerged in later discussions with the Swedish Chemical Agency that increasing concentrations of short-chain substances such as PFBS have been observed [84], and that such trends are of great concern. There is a need to find out whether this is a local or global trend and whether similar trends have been observed for additional PFASs with recently increased production volumes.

Another stakeholder that Mistra EviEM has consulted is the Swedish Agency for Marine and Water Management (SWAM), which is responsible for the Swedish environmental quality objectives "Flourishing Lakes and Streams" and "A Balanced Marine Environment, Flourishing Coastal Areas and Archipelagos". Even though SWAM's major interest in this topic is the toxic effects, the Agency is also interested in more information on sources, transportation processes and environmental fate of PFASs in general.

Since the consultations resulted in several conceivable review questions, the main stakeholders and a few scientists were invited to a discussion where the goal was to find a common ground and agree on the most relevant question. It was concluded that there is little or no controversy regarding the properties of PFCAs and PFSAs; they are in many cases persistent, bioaccumulative and toxic (PBT) and some of them are classified and treated as such. It is however less clear how new compounds with shorter carbon chains should be treated. Even though they may be toxic and extremely persistent they may not be bio-accumulative enough to be classified as PBT-compounds or vPvB compounds. The Swedish Chemicals Agency needs more information on the environmental fate of both phased-out and replacement compounds. To compile and evaluate that information is not necessarily an easy task since, e.g., the results in monitoring studies can appear to be somewhat contradictory. In some cases one particular compound has shown opposite temporal trends in different sample types even within the same region.

In order to make the review as relevant as possible to as many people as possible, a wider group of Swedish stakeholders were invited to discuss what the review should focus on. This group of stakeholders included governmental agencies, municipal drinking water producers, environmental consultants and NGOs. Some of the key points put forward at the meeting included

- $\quad$ PFOS and PFOA are the two most important phased-out substances, but their precursors are also important. Among replacement substances, the most toxic (if that is known) should be prioritized.

- Time trends in both biotic and abiotic samples should be included

- Contaminated areas as point sources are important to study. In what way will contaminated areas influence future time trends of concentrations in the surrounding environment?

- If time trends of replacement substances are scarce, the mere information on occurrences in the environment is also interesting.

The last point is out of the scope of this review and will not be considered. Additional questions that the stakeholders were seeking more information about included

- Are PFASs distributed differently between environmental media due to varying properties?

- How far should mitigation of contaminated areas be pushed? Is it possible to establish global or regional baseline or background concentrations?

- Can important knowledge gaps be identified?

One additional stakeholder that Mistra EviEM has consulted is the FluoroCouncil, which is a global organization representing a range of different fluorotechnology companies that manufacture, formulate or process fluoropolymer products, fluorotelomer-based products, fluoro-surfactants, and fluoro-surface property modification agents. The FluoroCouncil is involved in the 2010/2015 PFOA Stewardship Program, a global partnership between U.S. EPA and industry based on voluntary goals to eliminate PFOA from facility emissions and product content by 2015 .

\section{Objective of the review}

Although compounds such as PFOS and PFOA have been phased out in some parts of the world, for many reasons it cannot be taken for granted that this will lead to swift declines in environmental or human exposure. If new emissions of a PFAS were to cease, the high persistence of PFCAs and PFSAs may lead to delayed and very slow declines in exposure. In addition, existing environmental burdens of precursor substances (including fluorinated polymers) might continue to act as an indirect pseudo-source of PFCAs and PFSAs in the future, depending on their stability. Furthermore, continued production and use of these compounds in parts of the world where phase-out policies have yet to be implemented could influence human and environmental exposure in regions far from this production, owing to long-range transport or exporting of commercial products containing PFASs. An additional complication is 
the vast spatial heterogeneity of current PFCA and PFSA burdens in the world's oceans. For example, despite all recent regulatory actions (Figures 1 and 2), remote marine ecosystems in the Arctic are predicted to receive increased exposure to PFOS and PFOA in the future as ocean currents slowly transport relatively contaminated ocean water from mid-latitudes northwards to remote regions where current ocean concentrations are lower $[50,88,89]$. With all these complexities, uncertainties, and global spatial heterogeneity, it is difficult to predict the future of human and environmental exposure to PFASs. For this reason, an objective and systematic review of temporal trends reported in the literature was deemed a high priority.

In summary, PFCAs and PFSAs have unique environmental chemistry and much of the environmental fate of substances within these groups is still poorly understood. The objective of the proposed systematic review is to find out whether the concentrations of these substances in the environment are changing in any particular direction, and whether any spatial differences or changes in temporal concentration trends can be related to the implemented phase-outs. In addition, any temporal changes in the distribution of linear vs branched perfluoroalkyl chains for individual PFCAs and PFSAs will be explored to determine if isomer pattern changes can be associated with the phase-outs.

The review team has phrased the review question as "What is the effect of phasing out long-chain per-and polyfluoroalkyl substances on the concentrations of perfluoroalkyl acids and their precursors in the environment?" The question builds on the following elements:

- Population/Subject: abiotic and biological samples including general human populations.

- Intervention: Legislative or voluntary phase-out of production and use of long-chain PFASs

- Comparator: Before intervention

- Outcome: Change of concentrations of the phased-out substances and their precursors and substitutes.

\section{Methods \\ Searches}

Searches in literature databases will be made using the search terms displayed in Table 1. Using the Boolean operators indicated this translates into the search string below, where * is a wildcard that can be any number of characters, and a question mark is exactly one arbitrary character.

(perfluor* OR polyfluor* OR fluorotelomer* OR PF?S OR PF?A OR PFC OR PFT OR PFHxS OR FOSE OR FOSA OR PAPS) AND (((trend OR variation) NEAR (time OR temporal)) OR ((change OR increase OR decrease) NEAR/5 (level or concentration)) OR "time series" OR
Table 1 Suggested search strategy

\begin{tabular}{|c|c|c|c|}
\hline & Term 1 & AND & Term 2 \\
\hline & perfluor* & & $\begin{array}{l}\text { ((trend OR variation) NEAR (time OR } \\
\text { temporal)) }\end{array}$ \\
\hline OR & polyfluor* & OR & $\begin{array}{l}\text { ((change OR increase OR decrease) } \\
\text { NEAR/5 (level or concentration)) }\end{array}$ \\
\hline OR & fluorotelomer* & OR & "time series" \\
\hline OR & PF?S & OR & $\begin{array}{l}\text { ((snow OR ice OR sediment) NEAR } \\
\text { (core OR column OR cap)) }\end{array}$ \\
\hline OR & PF?A & OR & $\operatorname{archive}^{*}$ \\
\hline OR & PFC & OR & "specimen bank" \\
\hline OR & PFT & OR & "long-term monitoring" \\
\hline OR & PFHXS & OR & "repeated measurements" \\
\hline OR & FOSE & OR & historic $^{*}$ \\
\hline OR & FOSA & & \\
\hline OR & PAPS & & \\
\hline
\end{tabular}

Search terms are connected with the Boolean operator AND, and words within each term is connected by OR. Truncation is denoted by *. Question mark is a wildcard that represents exactly one character.

((snow OR ice OR sediment) NEAR (core OR column OR cap)) OR archive" OR "specimen bank" OR "long-term monitoring" OR "repeated measurements" OR historic")

The search string shown above and in Table 1 is designed for Web of Science. Other literature databases may use other wildcards or require less complex search strings. Adjusted search strings used in individual databases are shown in Additional file 2: Search strings used in individual literature databases. No particular constraints regarding time, document type, or language will be applied when searching for literature. At a later stage some languages may however be excluded due to limitations in translation resources. Literature databases that will be used for searching are listed in Table 2. The table also indicates the fields that will be searched and number of hits obtained in preliminary searches.

The comprehensiveness of the searches will be tested by cross-checking the hits with 1) a list of papers that the review team a priori think should be found by the searches, and 2) bibliographies in review articles. The list of papers that should be found is shown in Additional file 3: List of relevant articles that should be found in searches for literature.

In addition to data in the scientific literature it is anticipated that data will be found also in the grey literature. Such data will be searched for using the search engines Google and Google Scholar on the internet. For these search engines the search string below will be used. The same search string translated to Scandinavian languages and to German will also be used.

(pfas or pfaa or pfos or pfoa) (change or trend or temporal or increase or decrease). 
Table 2 Electronic databases used for searching

\begin{tabular}{|c|c|c|c|}
\hline Database & Searched field & No of hits ${ }^{3)}$ & Date \\
\hline ISI Web of Science ${ }^{1)}$ & topic & 3938 (3891) & $2014-05-13$ \\
\hline Science Direct & $\begin{array}{l}\text { title, abstract and } \\
\text { keywords }\end{array}$ & $3272(2869)$ & $2014-05-13$ \\
\hline Engineering Village ${ }^{2)}$ & $\begin{array}{l}\text { subject/title/ } \\
\text { abstract }\end{array}$ & $1217(728)$ & 2014-05-14 \\
\hline Scopus & $\begin{array}{l}\text { title, abstract and } \\
\text { keywords }\end{array}$ & $2502(639)$ & 2014-05-14 \\
\hline Academic search premier & $\begin{array}{l}\text { title/abstract/ } \\
\text { subject/keyword }\end{array}$ & $851(815)$ & 2014-05-14 \\
\hline Wiley Online Library & $\begin{array}{l}\text { abstract or title or } \\
\text { keywords }\end{array}$ & $641(548)$ & 2014-05-14 \\
\hline $\begin{array}{l}\text { Directory of Open } \\
\text { Access Journals }\end{array}$ & all fields & $42(39)$ & 2014-05-14 \\
\hline
\end{tabular}

${ }^{1)}$ Including Web of Science ${ }^{\text {Tm }}$ Core Collection, Medline ${ }^{\oplus}$ and SciELO

Citation Index.

${ }^{2)}$ Including Geobase and Georef.

${ }^{3)}$ Values in parentheses indicate number of items remaining after removal of duplicates when all databases are combined.

In addition, websites of relevant specialist organisations (listed below) will be searched. In this case the search strings will be website specific. All searches will however be reported in the systematic review. If no reports are found on the website, but the organisation still is thought to have relevant results, a letter will be sent to the appropriate person to try to obtain the information.

- Swedish Environmental Protection Agency (SEPA)

- US Environmental Protection Agency (USEPA)

- FluoroCouncil

- Society of the Plastics Industry

- Arctic Monitoring and Assessment Programme (AMAP)

- Danish Environmental Protection Agency

- Norwegian Environment Agency

- Finnish Environment Institute (SYKE)

- German Umweltsbundesamt

- UK Environment Agency

- HELCOM

- EEA

- Stockholm Convention GMP report to COP

- Netherlands RIVM

- National food agencies

- Environment Canada

- Canadian Northern Contaminants Program

- US CDC, National Health and Nutrition Examination Survey (NHANES)

- Other relevant organisations we identify

The reviewers will also send a standardized email to their scientific networks asking for information on possible studies that are soon to be available or in the grey literature.

\section{Study inclusion criteria}

The interventions relevant for the systematic review (phase-outs of long-chain PFASs) are intended to have a global rather than a local impact, and therefore the concentrations at one location may be impacted by a combination of more than one phase-out. Consequently, measured concentrations are in most studies not directly related to any particular phase-out. In fact, useful time trends may even be found in studies where the purpose of the study was completely unrelated to any phase-out of PFASs at all. This means that studies qualified for the systematic review will not be restricted to intervention studies, but will potentially include any study reporting on at least one temporal PFAS concentration trend. Since it is known when and where the interventions have been implemented it may be possible to relate these to the changes in concentrations, and hence, intervention should still be a justified question element.

Relevant temporal trends may be obtained by recurring measurements at a given location (monitoring) or by means of environmental archives (e.g. dated sediment cores, ice cores) or specimen banks (e.g. biota, human diet, human samples). There will be no geographic limitations. However, to be included in the systematic review, the articles must pass each of the following relevance criteria.

- Relevant population or subject: abiotic and biological samples, including general human populations, exposed to ambient loads of PFASs and their precursors. Populations with occupational exposures related to manufacturing of PFASs, or with deliberate exposures in controlled trials, will be excluded.

- Types of outcome: time trends in concentrations of PFASs covering at least two years. Ideally the studies should provide concentration data from both before and after an intervention. In that case it is possible to compare the concentrations before and after the intervention. This is however not a prerequisite for inclusion in the systematic review since there are other means of evaluating temporal trends (see section Data synthesis and presentation).

At the title and abstract level all retrieved articles will be screened by two reviewers. To check that the screening is consistent and complies with the agreed inclusion/ exclusion criteria, a small subset (10\%) of the retrieved articles will also be double screened by the other reviewers. In case it cannot be decided whether the article should be included or excluded on the title and abstract level, the article will pass to the full text level. To evaluate the consistency of the screening, Kappa tests will be used. When screening at the full-text level the articles 
will be screened in the same manner as at the title and abstract level. A subset of at least $10 \%$ of the articles will be double screened. Again, Kappa tests will be used to test the consistency between the reviewers. If any Kappa test shows unacceptable discrepancies $(\kappa<0.6)$ the inclusion/exclusion criteria will be revisited by all reviewers and defined in a more unambiguous way. At both title/ abstract and full text levels, excluded articles will be coded with a reason for exclusion. A list of all articles excluded at full text screening, with reasons for exclusion, will be provided in the systematic review.

\section{Potential effect modifiers and reasons for heterogeneity}

The outcome may to a large extent be a function of time, and one obvious factor influencing the outcome is the timing of the study relative to the interventions. A study with data from just a few years directly after the intervention is likely to show a smaller effect than a more recent study covering a longer study period.

Although the interventions are intended to have a global impact, the outcome may for several reasons vary considerably depending on the location of the studies. Since the interventions are not yet implemented globally, it is reasonable to assume that the effect will be smaller in regions close to present sources compared to regions close to past sources where the interventions have been implemented.

Contaminated areas may also be a significant factor. In areas where the phase-out has been implemented, contaminated areas may still be present and leaching the phased-out substances to the surrounding environments.

In more remote regions, such as the Arctic, the outcome may depend on the predominating mode of transport. In terrestrial or high-altitude areas where the PFAS source is dominated by long-range transport of volatile precursors, the response to the interventions may be quicker compared to coastal areas where the PFAS source is dominated by direct long-range transport in the aquatic environment. The measured outcome may also depend on sample type.

Food web dynamics, e.g. temporal shifts in diet and/or growth rates over the time-scale of decades, can influence long-term temporal trends of persistent organic contaminants. For example, in the northern Baltic Sea, herring have been shown over the long term to grow more slowly which is related to shift in feeding at one trophic level higher than they did in the past. Bioenergetics modelling undertaken by Peltonen et al. [90] suggests that changes in the feeding behaviour of herring can explain the "levelling off" of the downward temporal trends of polychlorinated dibenzo-p-dioxins and polychlorinated dibenzofurans (PCDD/Fs) in herring from the northern Baltic Sea [91]. Among studies of human samples, the results may vary between cross-sectional studies, where different people are studied every year, and prospective/longitudinal studies, where the same individuals are followed over time.

Analytical quality may also be a reason for heterogeneity. In the early years standards were not always available for accurate calibration of the analytical instruments. Both accuracy and precision have been improved in recent years, and the detection limits for PFASs were in general 100 times higher in 2000 than in 2014. The ability to distinguish between linear and branched perfluroalkyl chains and to analyse a wider range of substances have helped to improve our understanding of heterogeneities between studies.

In summary, potential effect modifiers and reasons for heterogeneity may include:

Timing of the study relative to the interventions

Proximity to past and present sources

Geographical differences

Mode of predominant transport

Type of sample e.g. sampling method, matrix, individual vs pooled

For human data, design of study (e.g. cross-sectional, prospective, age, sex)

Analytical quality in relation to time of the study (e.g. availability of standards)

Food web changes over time

Species differences

\section{Study quality assessment}

During critical appraisal of relevant studies, information that directly affects the internal validity of each study will be recorded in a pre-designed Excel spreadsheet. The assessment of the internal validity will focus on the following aspects:

- Selection bias. In this case the comparison groups are formed by samples taken at different times. The information about samples and what they represent must be sufficient to determine whether samples from different sampling occasions are comparable.

- Dating of samples. In most cases it is known when samples are collected, but samples from environmental archives such as sediment or ice cores are more complicated to date accurately. Dating by means of e.g. isotope techniques that give an absolute age to each individual sample may be regarded as high quality dating. Dating by means of historical markers (e.g. peak concentrations of other contaminants) providing relative ages may be regarded as acceptable dating. Post-depositional perturbations caused by for instance thawing-freezing cycles in snow or bioturbation in sediments should be discussed and assessed. 
- Sample integrity. Are sample pre-treatment, sample preservation, prevention of contamination and storage methods suitable for the sample type?

- Analytical quality. Are analytical procedures appropriate? Did instrumentation, internal standards, procedural blanks, LOD/LOQ or field blanks change over time? If so, what is the risk that these parameters have influenced the measured temporal trends?

Study length and sampling frequency will be recorded but will generally not be part of the regular quality assessment since these parameters presumably will be reflected by the standard errors and confidence intervals of the effect sizes in each study. Study length and sampling frequency will thus affect the weighting of each study in the quantitative synthesis. The number of individual or pooled samples will also be recorded. Standard errors are likely to be smaller in studies with multiple and individually analysed samples at each sampling occasion than studies with just one sample from each time point. A high number of pooled samples at each sampling occasion may increase the chance of obtaining results representative for these time points and may therefore decrease the scatter along a regression line. In this way the number of pooled samples is also likely to affect the weighting of each study in the quantitative synthesis.

Information important for the assessment of external validity, or how transferable the studies are to the context of the question, will be recorded as well. As indicated by the study inclusion criteria, relatively few restrictions regarding populations/subjects and geographical locations will be applied. The external validity is therefore mainly related to subgroup analysis that will be performed. Information about environmental setting and important sample covariates should be reported to such an extent that each study confidently can be grouped with other studies or be judged to stand alone. Also, the study should cover a time period that is possible to relate to any known relevant phase-out.

Relevant studies that are judged to have low risk for selection bias, have accurately dated samples, ensured good sample integrity, have used appropriate analytical procedures, and provide sufficient information regarding external validity will meet the quality criteria and hence be included in the quantitative synthesis. Any reason for not including a study based on quality will be recorded in the data spreadsheet and a list of all articles excluded during critical appraisal, with reasons for exclusion, will be presented in the systematic review. However, studies that fail to qualify for the quantitative synthesis will be used in sensitivity analyses where it will be tested if the inclusion or exclusion of these studies influence the overall results.
Critical appraisal of relevant studies will be carried out by four of the reviewers. To ensure a high consistency between the reviewers at least $25 \%$ of the relevant articles will be double-checked and Kappa tests will be used to test the consistency. In addition to this, any articles that for some reason are difficult to critically appraise will be discussed by the entire review team before any decisions are made.

\section{Data extraction strategy}

Data (concentrations and other important study information) will be extracted from relevant studies and recorded in pre-designed Excel data sheets. Information directly relevant to the internal validity of the studies will be extracted and recorded by the reviewers that carry out the critical appraisal, while the rest of the data will be extracted and recorded by two other reviewers. However, the design of the data extraction sheets and how they should be filled will be approved in advance by all reviewers, and double-checking by all reviewers will be done for at least $25 \%$ of the articles. Data will always be recorded as reported in the primary studies. All necessary transformations and calculations will be performed at the analysis stage.

The outcome data that will be recorded is the PFAS concentrations in the studied samples at different times and/or slopes of trends during specified time periods. The location of these data within each article, as well as an indication whether the data have been graphically extracted from figures, will be recorded. The types of data that will be compiled include sample type (air, sediment, species etc.), matrix (filter, $2 \mathrm{~cm}$ slice, liver, dissolved vs total water etc.), number of pooled or individual samples, covariates (sampling time, age, sex etc.), statistical variates (concentrations, slope of trend, linear-non-linear etc.), geographical location, time period covered, number of years covered. Data extraction may include contact with individual scientists for complementary information or for raw data or unpublished data.

\section{Data synthesis and presentation}

Meta-analyses for each PFAS will be performed using random effect models [92]. One possible effect size will be based on average concentrations during specified periods before and after the implementation of an intervention, respectively. All studies do however not use the same scale. Different units for concentration are used for different sample types (e.g. water and sediment), and different sample types show different concentration levels depending on the partition coefficient between the sample types. Different units for concentration may also be used for a certain sample type. For example, the concentrations in sediment samples are sometimes given as $\mathrm{ng} / \mathrm{g}$ bulk sediment and sometimes as ng/g TOC (Total 
Organic Carbon). All of this means that raw differences in average concentrations cannot be used as effect size. Therefore response ratios will be used as the effect size. The response ratio $\mathrm{R}$ is calculated according to equation 1 , where $\bar{C}_{0}$ and $\bar{C}_{I}$ are the average concentration during a specified period before and after the implementation of the intervention, respectively.

$$
R=\bar{C}_{I}
$$

Another effect size that will be explored is the normalized rate of change in concentration. It is possible that even though there is no significant difference in average concentrations, there can be a significant trend in some direction after the intervention. Moreover, although the evidence for a causal relationship between the outcome and the intervention will be weaker, the rate of change in concentration can be evaluated when pre-intervention data is missing or when more than one intervention influence the outcome. This means that also nonintervention studies can be included. The rate of change will be calculated by means of regression analyses. Again, since different scales are used in different studies, the rate of change needs to be normalized. If the rate of change is normalized to the average concentration before the intervention the effect size $C^{\prime}$ is calculated as

$$
C^{\prime}=\frac{1}{\bar{C}_{0}} \cdot \frac{\partial C}{\partial t}
$$

where $C_{0}$ is concentration at time $\mathrm{t}=0$. It may also be possible to compare the normalised rates of change in concentration before and after the intervention, respectively, if that data exist.

Obviously, with such a wide range of subjects or populations that potentially can be included in the systematic review it will be necessary to perform subgroup analyses. It is envisaged that for each PFAS analysed, the splitting into subgroups will be based on e.g. sample type, species and geographical region. A narrative synthesis will be prepared using tables of study characteristics and results. Visualisation of results may be done using forest plots and other graphical representations.

\section{Additional files}

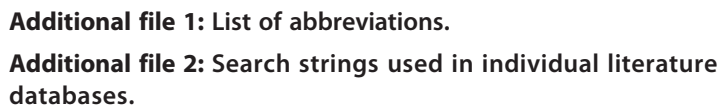

\section{Authors' contributions}

This systematic review protocol is based on a draft written by ML. All authors discussed the draft and suggested improvements. The major part of the background section is written by JWM, ITC and DH. All authors read and approved the final manuscript.

\section{Acknowledgements}

The preparation of this protocol and the forthcoming review is financed by the Mistra Council for Evidence-based Environmental Management (Mistra EviEM). EviEM is funded by the Swedish Foundation for Strategic Environmental Research (Mistra) and hosted by the Royal Swedish Academy of Sciences. The authors wish to thank two anonymous reviewers for their insightful comments. We are also grateful for the comments made by stakeholders during a public review of an earlier version of this protocol.

\section{Author details}

${ }^{1}$ Mistra EviEM, The Royal Swedish Academy of Sciences, Box 50005, SE-104 05 Stockholm, Sweden. ${ }^{2}$ Department of Environmental Science and Analytical Chemistry (ACES), Stockholm University, SE-106 91 Stockholm, Sweden. ${ }^{3}$ Norwegian Inst Air Res, FRAM High North Res Ctr Climate \& Environm, Tromso, Norway. ${ }^{4}$ Division of Analytical and Environmental Toxicology, 10-102C Clinical Sciences, Edmonton, Alberta T6G 2G3, Canada.

Received: 2 September 2014 Accepted: 24 November 2014

Published: 27 January 2015

\section{References}

1. Lindstrom AB, Strynar MJ, Libelo EL. Polyfluorinated compounds: past, present, and future. Environ Sci Technol. 2011;45:7954-61.

2. Kissa E. Fluorinated Surfactants and Repellents. 2nd ed. New York (NY): Marcel Dekker, Inc.; 2001

3. Prevedouros K, Cousins IT, Buck RC, Korzeniowski SH. Sources, fate and transport of perfluorocarboxylates. Environ Sci Technol. 2006;40:32-44.

4. Wang Z, Cousins IT, Scheringer M, Buck RC, Hungerbühler K. Global emission inventories for C4-C14 perfluoroalkyl carboxylic acid (PFCA) homologues from 1951 to 2030, Part I: production and emissions from quantifiable sources. Environ Int. 2014;70:62-75.

5. Wang Z, Cousins IT, Scheringer M, Buck RC, Hungerbühler K. Global emission inventories for C4-C14 perfluoroalkyl carboxylic acid (PFCA) homologues from 1951 to 2030, part II: the remaining pieces of the puzzle. Environ Int. 2014;69:166-76.

6. Paul AG, Jones KC, Sweetman AJ. A first global production, emission, and environmental inventory for perfluorooctane sulfonate. Environ Sci Technol. 2009:43:386-92.

7. Armitage JM, MacLeod M, Cousins IT. Modeling the Global Fate and Transport of Perfluorooctanoic Acid (PFOA) and Perfluorooctanoate (PFO) Emitted from direct sources using a multispecies mass balance model. Environ Sci Technol. 2009;43:1134-40.

8. Smart BE, Dixon DA. Bond-energies and stabilities of poly(perfluoroethers). Abstr Pap Am Chem Soc. 1994;207:31-FLUO.

9. Buck RC, Franklin J, Berger U, Conder JM, Cousins IT, de Voogt P, et al. Perfluoroalkyl and polyfluoroalkyl substances in the environment: terminology, classification, and origins. Integr Environ Assess Manag. 2011;7:513-41.

10. Becker AM, Gerstmann S, Frank H. Perfluorooctane surfactants in waste waters, the major source of river pollution. Chemosphere. 2008;72:115-21.

11. Li XY, Zhang PY, Jin L, Shao T, Li ZM, Cao JJ. Efficient photocatalytic decomposition of perfluorooctanoic acid by indium oxide and its mechanism. Environ Sci Technol. 2012;46:5528-34.

12. Igarashi S, Yotsuyanagi T. Homogeneous liquid-liquid-extraction by ph dependent phase-separation with a fluorocarbon ionic surfactant and its application to the preconcentration of porphyrin compounds. Mikrochim Acta. 1992;106:37-44.

13. Lopez-Fontan $J \mathrm{~L}$, Sarmiento F, Schulz PC. The aggregation of sodium perfluorooctanoate in water. Colloid Polym Sci. 2005;283:862-71.

14. Wang ZY, MacLeod M, Cousins IT, Scheringer M, Hungerbuhler K. Using COSMOtherm to predict physicochemical properties of poly- and perfluorinated alkyl substances (PFASs). Environ Chem. 2011;8:389-98.

15. Vierke L, Berger U, Cousins IT. Estimation of the acid dissociation constant of perfluoroalkyl carboxylic acids through an experimental investigation of their water-to-air transport. Environ Sci Technol. 2013;47:11032-9. 
16. Higgins $C P$, Luthy RG. Sorption of perfluorinated surfactants on sediments. Environ Sci Technol. 2006:40:7251-6.

17. Gottschall N, Topp E, Edwards M, Russell P, Payne M, Kleywegt S, et al. Polybrominated diphenyl ethers, perfluorinated alkylated substances, and metals in tile drainage and groundwater following applications of municipal biosolids to agricultural fields. Sci Total Environ. 2010;408:873-83.

18. Ahrens L, Yeung LWY, Taniyasu S, Lam PKS, Yamashita N. Partitioning of perfluorooctanoate (PFOA), perfluorooctane sulfonate (PFOS) and perfluorooctane sulfonamide (PFOSA) between water and sediment. Chemosphere. 2011:85:731-7.

19. Benskin JP, Ahrens L, Muir DCG, Scott BF, Spencer C, Rosenberg B, et al. Manufacturing Origin of Perfluorooctanoate (PFOA) in Atlantic and Canadian Arctic Seawater. Environ Sci Technol. 2012:46:677-85.

20. Yamashita N, Taniyasu S, Petrick G, Wei S, Gamo T, Lam PKS, et al. Perfluorinated acids as novel chemical tracers of global circulation of ocean waters. Chemosphere. 2008;70:1247-55.

21. Barton CA, Zarzecki CJ, Russell MH. A site-specific screening comparison of modeled and monitored air dispersion and deposition for perfluorooctanoate. J Air Waste Manage Assoc. 2010;60:402-11.

22. Ellis DA, Martin JW, De Silva AO, Mabury SA, Hurley MD, Sulbaek Andersen MP, et al. Degradation of fluorotelomer alcohols: a likely atmospheric source of perfluorinated carboxylic acids. Environ Sci Technol. 2004;38:3316-21.

23. Armitage JM, MacLeod M, Cousins IT. Comparative Assessment of the Global Fate and Transport Pathways of Long-Chain Perfluorocarboxylic Acids (PFCAs) and Perfluorocarboxylates (PFCs) Emitted from Direct Sources. Environ Sci Technol. 2009;43:5830-6.

24. EFSA. Opinion of the Scientific Panel on Contaminants in the Food chain on Perfluorooctane sulfonate (PFOS), perfluorooctanoic acid (PFOA) and their salts. FSA J. 2008:653:1-131.

25. Ahrens L, Herzke D, Huber S, Bustnes JO, Bangjord G, Ebinghaus R. Temporal trends and pattern of polyfluoroalkyl compounds in tawny owl (Strix aluco) eggs from Norway, 1986-2009. Environ Sci Technol. 2011;45:8090-7.

26. Holmstrom KE, Johansson AK, Bignert A, Lindberg P, Berger U. Temporal trends of perfluorinated surfactants in swedish peregrine falcon eggs (Falco peregrinus), 1974-2007. Environ Sci Technol. 2010;44:4083-8.

27. Vicente J, Bertolero A, Meyer J, Viana P, Lacorte S. Distribution of perfluorinated compounds in Yellow-legged gull eggs (Larus michahellis) from the Iberian Peninsula. Sci Total Environ. 2012;416:468-75.

28. Houde M, De Silva AO, Muir DCG, Letcher RJ. Monitoring of perfluorinated compounds in aquatic biota: an updated review PFCs in aquatic biota. Environ Sci Technol. 2011;45:7962-73.

29. Lindh CH, Rylander L, Toft G, Axmon A, Rignell-Hydbom A, Giwercman A, et al. Blood serum concentrations of perfluorinated compounds in men from Greenlandic Inuit and European populations. Chemosphere. 2012;88:1269-75.

30. Sturm R, Ahrens L. Trends of polyfluoroalkyl compounds in marine biota and in humans. Environ Chem. 2010;7:457-84.

31. Conder JM, Hoke RA, De Wolf W, Russell MH, Buck RC. Are PFCAs bioaccumulative? A critical review and comparison with regulatory lipophilic compounds. Environ Sci Technol. 2008:42:995-1003.

32. Tomy GT, Pleskach K, Ferguson SH, Hare J, Stern G, Macinnis G, et al. Trophodynamics of some PFCs and BFRs in a Western Canadian Arctic Marine Food Web. Environ Sci Technol. 2009;43:4076-81.

33. Kelly BC, Ikonomou MG, Blair JD, Surridge B, Hoover D, Grace R, et al. Perfluoroalkyl contaminants in an arctic marine food web: trophic magnification and wildlife exposure. Environ Sci Technol. 2009;43:4037-43.

34. Loi ElH, Yeung LWY, Taniyasu S, Lam PKS, Kannan K, Yamashita N. Trophic magnification of poly- and perfluorinated compounds in a subtropical food web. Environ Sci Technol. 2011;45:5506-13.

35. Martin JW, Whittle DM, Muir DCG, Mabury SA. Perfluoroalkyl contaminants in a food web from lake Ontario. Environ Sci Technol. 2004;38:5379-85.

36. Muller CE, De Silva AO, Small J, Williamson M, Wang XW, Morris A, et al. Biomagnification of perfluorinated compounds in a remote terrestrial food chain: lichen-caribou-wolf. Environ Sci Technol. 2011;45:8665-73.

37. Russell MH, Nilsson H, Buck RC. Elimination kinetics of perfluorohexanoic acid in humans and comparison with mouse, rat and monkey. Chemosphere. 2013;93:2419-25.

38. Du GZ, Huang HY, Hu JL, Qin YF, Wu D, Song L, et al. Endocrine-related effects of perfluorooctanoic acid (PFOA) in zebrafish, H295R steroidogenesis and receptor reporter gene assays. Chemosphere. 2013;91:1099-106.
39. Joensen UN, Veyrand $B$, Antignac JP, Jensen MB, Petersen JH, Marchand $P$, et al. PFOS (perfluorooctanesulfonate) in serum is negatively associated with testosterone levels, but not with semen quality, in healthy men. Hum Reprod. 2013;28:599-608.

40. Kjeldsen LS, Bonefeld-Jorgensen EC. Perfluorinated compounds affect the function of sex hormone receptors. Environ Sci Pollut Res. 2013;20:8031-44.

41. Ikeda T, Aiba K, Fukuda K, Tanaka M. The induction of peroxisome proliferation in rat-liver by perfluorinated fatty-acids, metabolically inert derivatives of fatty-acids. J Biochem. 1985;98:475-82.

42. Kudo N, Bandai N, Suzuki E, Katakura M, Kawashima Y. Induction by perfluorinated fatty acids with different carbon chain length of peroxisomal beta-oxidation in the liver of rats. Chem Biol Interact. 2000;124:119-32.

43. Upham BL, Deocampo ND, Wurl B, Trosko JE. Inhibition of gap junctional intercellular communication by perfluorinated fatty acids is dependent on the chain length of the fluorinated tail. Int J Cancer. 1998;78:491-5.

44. Vanden Heuvel JP. Perfluorodecanoic acid as a useful pharmacologic tool for the study of peroxisome proliferation. Gen Pharmacol Vasc Syst. 1996;27:1123-9.

45. 3M. Letter to US EPA Re: phase-out plan for POSF-based products (226-0600). US EPA Adm Rec. 2000;226:1-11.

46. 2010/2015 PFOA Stewardship Programme. http://www.epa.gov/oppt/pfoa/ pubs/stewardship/index.html.

47. ECHA. Candidate list of substances of very high concern for authorisation. 2014; http://echa.europa.eu/web/guest/candidate-list-table.

48. Ritter S. Fluorochemicals go short. Chem Eng News. 2010;88:12-7.

49. Wang ZY, Cousins IT, Scheringer M, Hungerbuhler K. Fluorinated alternatives to long-chain perfluoroalkyl carboxylic acids (PFCAs), perfluoroalkane sulfonic acids (PFSAs) and their potential precursors. Environ Int. 2013:60:242-8

50. Armitage JM, Schenker U, Scheringer M, Martin JW, MacLeod M, Cousins IT. Modeling the global fate and transport of perfluorooctane sulfonate (PFOS) and precursor compounds in relation to temporal trends in wildlife exposure. Environ Sci Technol. 2009;43:9274-80.

51. Martin JW, Asher BJ, Beesoon S, Benskin JP, Ross MS. PFOS or PreFOS? Are perfluorooctane sulfonate precursors (PreFOS) important determinants of human and environmental perfluorooctane sulfonate (PFOS) exposure? J Environ Monit. 2010;12:1979-2004.

52. Benskin JP, Muir DCG, Scott BF, Spencer C, De Silva AO, Kylin H, et al. Perfluoroalkyl acids in the Atlantic and Canadian Arctic Oceans. Environ Sci Technol. 2012:46:5815-23.

53. Filipovic M, Berger U, McLachlan MS. Mass balance of perfluoroalkyl acids in the Baltic sea. Environ Sci Technol. 2013;47:4088-95.

54. Hu JY, Yu J, Tanaka S, Fujii S. Perfluorooctane Sulfonate (PFOS) and Perfluorooctanoic Acid (PFOA) in water environment of Singapore. Water Air Soil Pollut. 2011;216:179-91.

55. Kwok KY, Yamazaki E, Yamashita N, Taniyasu S, Murphy MB, Horii Y, et al. Transport of Perfluoroalkyl substances (PFAS) from an arctic glacier to downstream locations: implications for sources. Sci Total Environ. 2013:447:46-55

56. Wang X, Halsall C, Codling G, Xie Z, Xu B, Zhao Z, et al. Accumulation of perfluoroalkyl compounds in tibetan mountain snow: temporal patterns from 1980 to 2010. Environ Sci Technol. 2014;48:173-81.

57. Ahrens L. Polyfluoroalkyl compounds in the aquatic environment: a review of their occurrence and fate. J Environ Monit. 2011;13:20-31.

58. Braune BM, Letcher RJ. Perfluorinated sulfonate and carboxylate compounds in eggs of seabirds breeding in the Canadian Arctic: temporal trends (1975-2011) and interspecies comparison. Environ Sci Technol. 2013:47:616-24.

59. Giesy JP, Kannan K. Global distribution of perfluorooctane sulfonate in wildlife. Environ Sci Technol. 2001;35:1339-42.

60. Hart K, Gill VA, Kannan K. Temporal trends (1992-2007) of perfluorinated chemicals in northern sea otters (Enhydra lutris kenyoni) from South-Central Alaska. Arch Environ Contam Toxicol. 2009;56:607-14.

61. Lofstrand K, Jorundsdottir H, Tomy G, Svavarsson J, Weihe P, Nygard T, et al. Spatial trends of polyfluorinated compounds in guillemot (Uria aalge) eggs from North-Western Europe. Chemosphere. 2008;72:1475-80.

62. Shi $Y L$, Pan $Y Y$, Yang RQ, Wang $Y W$, Cai $Y Q$. Occurrence of perfluorinated compounds in fish from Qinghai-Tibetan Plateau. Environ Int. 2010;36:46-50.

63. Calafat AM, Kuklenyik Z, Caudill SP, Reidy JA, Needham LL. Perfluorochemicals in pooled serum samples from United States residents in 2001 and 2002. Environ Sci Technol. 2006;40:2128-34. 
64. Chen CL, Lu YL, Zhang X, Geng J, Wang TY, Shi YJ, et al. A review of spatial and temporal assessment of PFOS and PFOA contamination in China. Chem Ecol. 2009;25:163-77.

65. Ericson I, Gomez M, Nadal M, van Bavel B, Lindstrom G, Domingo JL. Perfluorinated chemicals in blood of residents in Catalonia (Spain) in relation to age and gender: A pilot study. Environ Int. 2007:33:616-23.

66. Fromme H, Midasch O, Twardella D, Angerer J, Boehmer S, Liebl B. Occurrence of perfluorinated substances in an adult German population in southern Bavaria. Int Arch Occup Environ Health. 2007;80:313-9.

67. Ji K, Kim S, Kho Y, Paek D, Sakong J, Ha J, et al. Serum concentrations of major perfluorinated compounds among the general population in Korea: dietary sources and potential impact on thyroid hormones. Environ Int. 2012:45:78-85

68. Karrman A, Domingo JL, Llebaria X, Nadal M, Bigas E, van Bavel B, et al. Biomonitoring perfluorinated compounds in Catalonia, Spain: concentrations and trends in human liver and milk samples. Environ Sci Pollut Res. 2010;17:750-8.

69. Kato K, Calafat AM, Wong LY, Wanigatunga AA, Caudill SP, Needham LL. Polyfluoroalkyl compounds in pooled sera from children participating in the national health and nutrition examination survey 2001-2002. Environ Sci Technol. 2009;43:2641-7.

70. Volkel W, Genzel-Boroviczeny O, Demmelmair H, Gebauer C, Koletzko B, Twardella D, et al. Perfluorooctane sulphonate (PFOS) and perfluorooctanoic acid (PFOA) in human breast milk: results of a pilot study. Int J Hyg Environ Health. 2008;211:440-6

71. Fernandez-Sanjuan M, Faria M, Lacorte S, Barata C. Bioaccumulation and effects of perfluorinated compounds (PFCs) in zebra mussels (Dreissena polymorpha). Environ Sci Pollut Res. 2013;20:2661-9.

72. Lasier PJ, Washington JW, Hassan SM, Jenkins TM. Perfluorinated chemicals in surface waters and sediments from northwest georgia, usa, and their bioaccumulation in lumbriculus variegatus. Environ Toxicol Chem. 2011:30:2194-201.

73. Fang S, Chen X, Zhao S, Zhang Y, Jiang W, Yang L, et al. Trophic magnification and isomer fractionation of perfluoroalkyl substances in the food web of Taihu Lake, China. Environ Sci Technol. 2014;48:2173-82.

74. Kannan K, Tao L, Sinclair E, Pastva SD, Jude DJ, Giesy JP. Perfluorinated compounds in aquatic organisms at various trophic levels in a Great Lakes food chain. Arch Environ Contam Toxicol. 2005;48:559-66.

75. Martin JW, Mabury SA, Solomon KR, Muir DCG. Bioconcentration and tissue distribution of perfluorinated acids in rainbow trout (Oncorhynchus mykiss). Environ Toxicol Chem. 2003:22:196-204.

76. Martin JW, Mabury SA, Solomon KR, Muir DCG. Dietary accumulation of perfluorinated acids in juvenile rainbow trout (Oncorhynchus mykiss). Environ Toxicol Chem. 2003;22:189-95.

77. Zhao S, Zhu L, Liu L, Liu Z, Zhang Y. Bioaccumulation of perfluoroalkyl carboxylates (PFCAs) and perfluoroalkane sulfonates (PFSAs) by earthworms (Eisenia fetida) in soil. Environ Pollut. 2013;179:45-52.

78. Olsen GW, Mair DC, Church TR, Ellefson ME, Reagen WK, Boyd TM, et al. Decline in perfluorooctanesulfonate and other polyfluoroalkyl chemicals in American Red Cross adult blood donors, 2000-2006. Environ Sci Technol. 2008:42:4989-95.

79. Zhang L, Liu JG, Hu JX, Liu C, Guo WG, Wang Q, et al. The inventory of sources, environmental releases and risk assessment for perfluorooctane sulfonate in China. Environ Pollut. 2012;165:193-8.

80. Renner R. The long and the short of perfluorinated replacements. Environ Sci Technol. 2006:40:12-3.

81. Kirchgeorg T, Weinberg I, Dreyer A, Ebinghaus R. Perfluorinated compounds in marine surface waters: data from the Baltic Sea and methodological challenges for future studies. Environ Chem. 2010;7:429-34.

82. Zhou Z, Liang Y, Shi Y, Xu L, Cai Y. Occurrence and transport of perfluoroalkyl acids (PFAAs), including short-chain PFAAs in Tangxun Lake, China. Environ Sci Technol. 2013;47:9249-57.

83. Persson S, Rotander A, Karrman A, van Bavel B, Magnusson U. Perfluoroalkyl acids in subarctic wild male mink (Neovison vison) in relation to age, season and geographical area. Environ Int. 2013;59:425-30.

84. Glynn A, Berger U, Bignert A, Ullah S, Aune M, Lignell S, et al. Perfluorinated alkyl acids in blood serum from primiparous women in Sweden: serial sampling during pregnancy and nursing, and temporal trends 1996-2010. Environ Sci Technol. 2012;46:9071-9.

85. The environmental objectives portal. http://www.miljomal.se/EnvironmentalObjectives-Portal/.
86. Borg D, Håkansson H. Environmental and Health Risk Assessment of Perfluoroalkylated and Polyfluoroalkylated Substances (PFASs) in Sweden. In: Book Environmental and Health Risk Assessment of Perfluoroalkylated and Polyfluoroalkylated Substances (PFASs) in Sweden (Editor ed.^eds.), vol. 6513. City: Swedish Environmental Protection Agency; 2012.

87. Molina ED, Balander R, Fitzgerald SD, Giesy JP, Kannan K, Mitchell R, et al. Effects of air cell injection of perfluorooctane sulfonate before incubation on development of the white leghorn chicken (Gallus domesticus) embryo. Environ Toxicol Chem. 2006;25:227-32.

88. Dietz R, Bossi R, Riget FF, Sonne C, Born EW. Increasing perfluoroalkyl contaminants in east Greenland polar bears (Ursus maritimus): a new toxic threat to the Arctic bears. Environ Sci Technol. 2008;42:2701-7.

89. Wania F. A global mass balance analysis of the source of perfluorocarboxylic acids in the Arctic ocean. Environ Sci Technol. 2007;41:4529-35.

90. Peltonen $\mathrm{H}$, Kiljunen $\mathrm{M}$, Kiviranta $\mathrm{H}$, Vuorinen PJ, Verta M, Karjalainen J. Predicting effects of exploitation rate on weight-at-age, population dynamics, and bioaccumulation of PCDD/Fs and PCBs in herring (Clupea harengus L.) in the Northern Baltic Sea. Environ Sci Technol. 2007:41:1849-55.

91. Miller A, Hedman JE, Nyberg E, Haglund P, Cousins IT, Wiberg K, et al. Temporal trends in dioxins (polychlorinated dibenzo-p-dioxin and dibenzofurans) and dioxin-like polychlorinated biphenyls in Baltic herring (Clupea harengus). Mar Pollut Bull. 2013;73:220-30.

92. Borenstein M, Hedges LV, Higgins JPT, Rothstein HR. Introduction to meta-analysis. John Wiley \& Sons, Ltd; 2009.

doi:10.1186/2047-2382-4-3

Cite this article as: Land et al:: What is the effect of phasing out long-chain per- and polyfluoroalkyl substances on the concentrations of perfluoroalkyl acids and their precursors in the environment? A systematic review protocol. Environmental Evidence 2015 4:3.

\section{Submit your next manuscript to BioMed Central and take full advantage of:}

- Convenient online submission

- Thorough peer review

- No space constraints or color figure charges

- Immediate publication on acceptance

- Inclusion in PubMed, CAS, Scopus and Google Scholar

- Research which is freely available for redistribution 